\title{
Existence of periodic solutions for two types of second-order nonlinear neutral integro-differential equations with infinite distributed mixed-delays
}

\author{
Hocine Gabsi ${ }^{\mathrm{a}}$, Abdelouaheb Ardjouni ${ }^{\mathrm{b}}$, Ahcene Djoudi ${ }^{\mathrm{c}}$ \\ ${ }^{a}$ Department of Mathematics, University of El-Oued, El-Oued, Algeria. \\ ${ }^{b}$ Department of Mathematics and Informatics, University of Souk Ahras, Souk Ahras, Algeria. \\ ${ }^{c}$ Department of Mathematics, University of Annaba, Annaba, Algeria.
}

\begin{abstract}
We consider two types of second-order neutral functional differential equations with infinite distributed delays and offer existence criteria for periodic solutions. During the process we invert the integro-differential equations into equivalent integral equations and derive suitable fixed point mappings. We show that these mappings fit into the framework of Schauder's fixed point theorem so that periodic solutions are readily obtained.
\end{abstract}

Keywords: Nonlinear neutral differential equations; periodic solutions; fixed point theorem; distributed delays.

2010 MSC: 34K13, 34A34, 34K40

\section{Introduction}

All biological systems and processes take time delays to complete. The delays can represent gestation times, incubation periods, or transport delays. In many cases time delays can be substantial such as gestation and maturation or can represent little lags such as acceleration and deceleration in physical processes. Therefore, it become natural to include time delay terms into the differential equations that model population

\footnotetext{
Email addresses: hocinegabsi@gmail.com (Hocine Gabsi), abd_ardjouni@yahoo.fr (Abdelouaheb Ardjouni), adjoudi@yahoo.com (Ahcene Djoudi)
} 
dynamics. Such models are referred as delay differential equation models. Thus, it seems clear that ordinary differential models are, at best, approximations of real word problems. In the last fifty years, delay models are becoming more common, appearing in many branches of biological, economical and physical modeling (see [1]-[12], [15]-[18]). This is due to their advantage of combining a simple, intuitive derivation with a wide variety of possible behavior regimes and to the fact that such models operate on an infinite dimensional space consisting of continuous functions that accommodate high dimensional dynamics (see [15]-[16]). More recently investigators have given special attentions to the study of equations in which the delay occurs in the derivative of the state variable as well as in the independent variable, so called neutral differential equations (see, [1]-[10], [12], [13], [17]). As known by Hale [15], Hale and Lunel [16], neutral delay differential equations appear as models of electrical networks which contain lossless transmission lines. Such networks arise, for example, in high speed computers where lossless transmission lines are used to interconnect switching circuits.

Existence and periodicity of solutions of functional differential equations are of great interest in mathematics and its applications to the modeling of various practical problems and have been extensively studied in recent times (see [1]-[13], [17]-[18] and references therein).

The study on neutral functional differential equations is more intricate than ordinary delay differential equations. This is why the studies of periodic solutions for neutral differential equations are relatively less than those devoted to ordinary differential equation. Most of the investigations on neutral type equations are confined to first order neutral differential equations. Very recently, Wu and Wang (see [18]) discussed the second order neutral delay differential equation

$$
(x(t)-c x(t-\delta))^{\prime \prime}+a(t) x(t)=\lambda b(t) f(x(t-\tau(t))),
$$

where $\lambda$ is a positive parameter, $\delta$ and $c$ are constants with $|c| \neq 1, a(\cdot), b(\cdot)$ are continuous positive functions, $f \in C([0, \infty),[0, \infty))$ and $a(\cdot), b(\cdot), \tau(\cdot)$ are periodic functions.

We consider the following two types of second-order neutral functional integro-differential equations with infinite distributed mixed-delays

$$
\begin{aligned}
& \frac{d^{2}}{d t^{2}}\left(x(t)-\int_{-\infty}^{t} f(t, s, x(s+t)) d s-\int_{t}^{\infty} g(t, s, x(s+t)) d s\right) \\
& =a(t) x(t)-b(t)\left(\int_{-\infty}^{t} p(t, s) h_{1}(x(s)) d s+\int_{t}^{\infty} q(t, s) h_{2}(x(s)) d s\right)
\end{aligned}
$$

and

$$
\begin{aligned}
& \frac{d^{2}}{d t^{2}}\left(x(t)-\int_{-\infty}^{t} f(t, s, x(s+t)) d s-\int_{t}^{\infty} g(t, s, x(s+t)) d s\right) \\
& =-a(t) x(t)+b(t)\left(\int_{-\infty}^{t} p(t, s) h_{1}(x(s)) d s+\int_{t}^{\infty} q(t, s) h_{2}(x(s)) d s\right) .
\end{aligned}
$$

Here $a, b \in C\left(\mathbb{R}, \mathbb{R}^{+}\right)$are $T$-periodic functions, $p: \triangle^{-} \rightarrow \mathbb{R}^{+}, q: \triangle^{+} \rightarrow \mathbb{R}^{+}, p(t+T, s+T)=p(t, s)$, $q(t+T, s+T)=q(t, s), f(t+T, s+T, x)=f(t, s, x)$ and $g(t+T, s+T, x)=g(t, s, x)$ with

$$
\triangle^{-}:=\{(t, s) \mid s \leq t\} \text { and } \triangle^{+}:=\{(t, s) \mid t \leq s\} .
$$

Also we assume that $f: \triangle^{-} \times \mathbb{R} \rightarrow \mathbb{R}^{+}, g: \triangle^{+} \times \mathbb{R} \rightarrow \mathbb{R}^{+}$and $h_{1}, h_{2}: \mathbb{R} \rightarrow \mathbb{R}$ are uniformly continuous functions at $x$.

Special cases of (1.1) and (1.2) have been considered and investigated by many authors. Particularly, W. Han and J. Ren in [17], Ardjouni et al. in [1], have, by choosing available operators and applying Krasnoselskii's fixed-point theorem, obtained sufficient conditions providing existence of periodic solutions to special cases of equations (1.1) and (1.2). 
The main features of this exposition are the following. In first section we introduce some notations and lemmas and state some preliminary results needed in later sections. Then we give the Green's function of (1.1) and (1.2), which plays an important role in our investigation. Also, we present the inversions of (1.1) and (1.2) and Schauder's fixed point theorem. For details on Schauder's theorem we refer the reader to [14]. In the last section, we present our main results on existence of periodic solutions of $(1.1)$ and $(1.2)$.

\section{Preliminaries}

For $T>0$, let $C_{T}$ be the set of all continuous scalar functions $x$ that are periodic in $t$ with period $T$. Then, endowed with the supremum norm,

$$
\|x\|:=\sup _{t \in \mathbb{R}}|x(t)|=\sup _{t \in[0, T]}|x(t)|,
$$

$\left(C_{T},\|\cdot\|\right)$ is a Banach space. Define

$$
C_{T}^{-}:=\left\{x \in C_{T} \mid x<0\right\}, C_{T}^{+}=\left\{x \in C_{T} \mid x>0\right\},
$$

and

$$
C_{T}^{J}:=\left\{\varphi \in C_{T} \mid\|\varphi\| \leq J\right\}
$$

Denote

$$
\begin{gathered}
M:=\sup \{a(t) \mid t \in[0, T]\}, m:=\inf \{a(t) \mid t \in[0, T]\}, \beta:=\sqrt{M}, \\
\lambda_{1}:=\frac{\exp \left(-\frac{\beta T}{2}\right)}{\beta(1-\exp (-\beta T))}, \mu_{1}:=\frac{1+\exp (-\beta T)}{2 \beta(1-\exp (-\beta T))},
\end{gathered}
$$

and

$$
\lambda_{2}:=\frac{\cos \left(\frac{\beta T}{2}\right)}{2 \beta \sin \left(\frac{\beta T}{2}\right)}, \mu_{2}:=\frac{1}{2 \beta \sin \left(\frac{\beta T}{2}\right)} .
$$

Throughout this section we let

$$
\begin{aligned}
& H_{1}(t, s, x):=b(t) p(t, s) h_{1}(x(s))-a(t) f(t, s, x(s+t)) \geq 0, \text { on } \triangle^{-} \times C_{T}^{J}, \\
& H_{2}(t, s, x):=b(t) q(t, s) h_{2}(x(s))-a(t) g(t, s, x(s+t)) \geq 0, \text { on } \triangle^{+} \times C_{T}^{J} .
\end{aligned}
$$

Also, in order to simplify notation, we define the function $H$ by

$$
H(t):=\int_{-\infty}^{t} H_{1}(t, s, x(s)) d s+\int_{t}^{\infty} H_{2}(t, s, x(s)) d s .
$$

Clearly, $H$ is a positive continuous function on $\mathbb{R}$.

Lemma 2.1 ([13]). The equation

$$
\frac{d^{2}}{d t^{2}} y(t)-M y(t)=h(t), h \in C_{T}^{-}
$$

has a unique T-periodic solution

$$
y(t)=\int_{t}^{t+T} K_{1}(t, s)(-h(s)) d s
$$

where

$$
K_{1}(t, s):=\frac{\exp (-\beta(s-t))+\exp (\beta(s-t-T))}{2 \beta(1-\exp (-\beta T))}, s \in[t, t+T] .
$$


Lemma $2.2([13]) . \lambda_{1} \leq K_{1}(t, s) \leq \mu_{1}$ and $\int_{t}^{t+T} K_{1}(t, s) d s=\frac{1}{M}$ for all $t \in[0, T]$ and $s \in[t, t+T]$.

Define the operator $P_{1}: C_{T} \rightarrow C_{T}$ by

$$
\left(P_{1} x\right)(t):=\left(I-T_{1} B_{1}\right)^{-1} T_{1}\left(\int_{-\infty}^{t}\left[-H_{1}(t, s, x)\right] d s+\int_{t}^{\infty}\left[-H_{2}(t, s, x)\right] d s\right),
$$

where

$$
\left(T_{1} h\right)(t)=\int_{t}^{t+T} K_{1}(t, s)(-h(s)) d s \text { and }\left(B_{1} y\right)(t)=[a(t)-M] y(t) .
$$

Lemma 2.3 ([13]). The equation

has a unique T-periodic solution

$$
\frac{d^{2}}{d t^{2}} y(t)-a(t) y(t)=h(t), h \in C_{T}^{-},
$$

$$
y(t)=\left(P_{1} h\right)(t)=\left(I-T_{1} B_{1}\right)^{-1} T_{1} h(t) .
$$

Lemma $2.4([13])$. $P_{1}$ satisfies

$$
0<\left(T_{1} h\right)(t) \leq\left(P_{1} h\right)(t) \leq \frac{M}{m}\left\|T_{1} h\right\|, h \in C_{T}^{-} .
$$

The following lemma is essential for our results on existence of periodic solution of (1.1).

Lemma 2.5. If $x \in C_{T}$, then $x$ is a solution of equation (1.1) if and only if

$$
x(t)=\int_{-\infty}^{t} f(t, s, x(s+t)) d s+\int_{t}^{\infty} g(t, s, x(s+t)) d s+\left(P_{1} x\right)(t),
$$

where $P_{1}$ is the map given by 2.1).

Proof. Let $x \in C_{T}$ be a solution of (1.1). Equation (1.1) can be rewritten as

$$
\begin{aligned}
& \frac{d^{2}}{d t^{2}}\left(x(t)-\int_{-\infty}^{t} f(t, s, x(s+t)) d s-\int_{t}^{\infty} g(t, s, x(s+t)) d s\right) \\
& -M\left(x(t)-\int_{-\infty}^{t} f(t, s, x(s+t)) d s-\int_{t}^{\infty} g(t, s, x(s+t)) d s\right) \\
& =[-M+a(t)]\left(x(t)-\int_{-\infty}^{t} f(t, s, x(s+t)) d s-\int_{t}^{\infty} g(t, s, x(s+t)) d s\right) \\
& +a(t)\left(\int_{-\infty}^{t} f(t, s, x(s+t)) d s+\int_{t}^{\infty} g(t, s, x(s+t)) d s\right) \\
& -b(t)\left(\int_{-\infty}^{t} p(t, s) h_{1}(x(s)) d s+\int_{t}^{\infty} q(t, s) h_{2}(x(s)) d s\right) .
\end{aligned}
$$

Taking

$$
y(t)=x(t)-\int_{-\infty}^{t} f(t, s, x(s+t)) d s-\int_{t}^{\infty} g(t, s, x(s+t)) d s .
$$

Then, 2.2 is transformed into

$$
\begin{aligned}
& \frac{d^{2}}{d t^{2}} y(t)-M y(t) \\
& =\left(B_{1} y\right)(t)+a(t)\left(\int_{-\infty}^{t} f(t, s, x(s+t)) d s+\int_{t}^{\infty} g(t, s, x(s+t)) d s\right) \\
& -b(t)\left(\int_{-\infty}^{t} p(t, s) h_{1}(x(s)) d s+\int_{t}^{\infty} q(t, s) h_{2}(x(s)) d s\right) \\
& =h(t) .
\end{aligned}
$$


From Lemma 2.1, we have

$$
\begin{aligned}
y(t) & =\left(T_{1} B_{1}\right) y(t) \\
& +T_{1}\left(\int_{-\infty}^{t} a(t) f(t, s, x(s+t)) d s+\int_{t}^{\infty} a(t) g(t, s, x(s+t)) d s\right) \\
& -T_{1}\left(\int_{-\infty}^{t} b(t) p(t, s) h_{1}(x(s)) d s+\int_{t}^{\infty} b(t) q(t, s) h_{2}(x(s)) d s\right) .
\end{aligned}
$$

This yields

$$
\left(I-T_{1} B_{1}\right) y(t)=T_{1}\left(\int_{-\infty}^{t}\left[-H_{1}(t, s, x)\right] d s+\int_{t}^{\infty}\left[-H_{2}(t, s, x)\right] d s\right) .
$$

Therefore, since $\left\|T_{1} B_{1}\right\| \leq 1-\frac{m}{M}<1$, we have

$$
y(t)=\left(I-T_{1} B_{1}\right)^{-1} T_{1}\left(\int_{-\infty}^{t}\left[-H_{1}(t, s, x)\right] d s+\int_{t}^{\infty}\left[-H_{2}(t, s, x)\right] d s\right) .
$$

Obviously

$$
\begin{aligned}
x(t) & =\int_{-\infty}^{t} f(t, s, x(s+t)) d s+\int_{t}^{\infty} g(t, s, x(s+t)) d s \\
& +\left(I-T_{1} B_{1}\right)^{-1} T_{1}\left(\int_{-\infty}^{t}\left[-H_{1}(t, s, x)\right] d s+\int_{t}^{\infty}\left[-H_{2}(t, s, x)\right] d s\right) .
\end{aligned}
$$

It is clear that $y(t)$ is the unique $T$-periodic solution of 2.3 for $h \in C_{T}^{-}$.

Lemma 2.6 ([13]). The equation

$$
\frac{d^{2}}{d t^{2}} y(t)+M y(t)=h(t), h \in C_{T}^{+},
$$

has a unique T-periodic solution

$$
y(t)=\int_{t}^{t+T} K_{2}(t, s) h(s) d s,
$$

where

$$
K_{2}(t, s)=\frac{\cos \beta\left(\frac{T}{2}+t-s\right)}{2 \beta \cos \frac{\beta T}{2}}, s \in[t, t+T] .
$$

Lemma 2.7 ([13]). $\int_{t}^{t+T} K_{2}(t, s) d s=\frac{1}{M}$. Furthermore, if $M<\left(\frac{\pi}{T}\right)^{2}$ then $\lambda_{2} \leq K_{2}(t, s) \leq \mu_{2}$ for all $t \in[0, T]$ and $s \in[t, t+T]$.

Next, define the operator $P_{2}: C_{T} \longrightarrow C_{T}$ by

$$
\left(P_{2} \varphi\right)(t):=\left(I-T_{2} B_{2}\right)^{-1} T_{2}\left(\int_{-\infty}^{t} H_{1}(t, s, \varphi(s)) d s+\int_{t}^{\infty} H_{2}(t, s, \varphi(s)) d s\right),
$$

where

$$
\left(T_{2} h\right)(t)=\int_{t}^{t+T} K_{2}(t, s) h(s) d s \text { and }\left(B_{2} y\right)(t)=[M-a(t)] y(t)
$$


Lemma 2.8 ([13]). The equation

has a unique T-periodic solution

$$
\frac{d^{2}}{d t^{2}} y(t)+a(t) y(t)=h(t), h \in C_{T}^{+},
$$

$$
y(t)=\left(P_{2} h\right)(t)=\left(I-T_{2} B_{2}\right)^{-1} T_{2} h(t) .
$$

Lemma 2.9 ([13]). If $M<\left(\frac{\pi}{T}\right)^{2}$ then

$$
0<\left(T_{2} h\right)(t) \leq\left(P_{2} h\right)(t) \leq \frac{M}{m}\left\|T_{2} h\right\|, h \in C_{T}^{+} .
$$

The following lemma is essential for our results on existence of periodic solution of 1.2 ).

Lemma 2.10. If $x \in C_{T}$ then $x$ is a solution of equation (1.2) if and only if

$$
x(t)=\int_{-\infty}^{t} f(t, s, x(s+t)) d s+\int_{t}^{\infty} g(t, s, x(s+t)) d s+\left(P_{2} x\right)(t),
$$

where $P_{2}$ is the map given by 2.4).

Proof. Let $x \in C_{T}$ be a solution of 1.2 . Equation 1.2 can be rewritten as

$$
\begin{aligned}
& \frac{d^{2}}{d t^{2}}\left(x(t)-\int_{-\infty}^{t} f(t, s, x(s+t)) d s-\int_{t}^{\infty} g(t, s, x(s+t)) d s\right) \\
& +M\left(x(t)-\int_{-\infty}^{t} f(t, s, x(s+t)) d s-\int_{t}^{\infty} g(t, s, x(s+t)) d s\right) \\
& =(M-a(t))\left(x(t)-\int_{-\infty}^{t} f(t, s, x(s+t)) d s-\int_{t}^{\infty} g(t, s, x(s+t)) d s\right) \\
& -a(t)\left(\int_{-\infty}^{t} f(t, s, x(s+t)) d s+\int_{t}^{\infty} g(t, s, x(s+t)) d s\right) \\
& +b(t)\left(\int_{-\infty}^{t} p(t, s) h_{1}(x(s)) d s+\int_{t}^{\infty} q(t, s) h_{2}(x(s)) d s\right) .
\end{aligned}
$$

Taking

$$
y(t)=x(t)-\int_{-\infty}^{t} f(t, s, x(s)) x(s) d s-\int_{t}^{\infty} g(t, s, x(s)) d s
$$

then $(2.5)$ is transformed into

$$
\begin{aligned}
& \frac{d^{2}}{d t^{2}} y(t)+M y(t) \\
& =\left(B_{2} y\right)(t)-a(t)\left(\int_{-\infty}^{t} f(t, s, x(s+t)) d s+\int_{t}^{\infty} g(t, s, x(s+t)) d s\right) \\
& +b(t)\left(\int_{-\infty}^{t} p(t, s) h_{1}(x(s)) d s+\int_{t}^{\infty} q(t, s) h_{2}(x(s)) d s\right) \\
& =h(t) .
\end{aligned}
$$

From Lemma 2.6, we have

$$
\begin{aligned}
y(t) & =\left(T_{2} B_{2}\right) y(t) \\
& -T_{2}\left(\int_{-\infty}^{t} a(t) f(t, s, x(s+t)) d s+\int_{t}^{\infty} a(t) g(t, s, x(s+t)) d s\right) \\
& +T_{2}\left(\int_{-\infty}^{t} b(t) p(t, s) h_{1}(x(s)) d s+\int_{t}^{\infty} b(t) q(t, s) h_{2}(x(s)) d s\right) .
\end{aligned}
$$


This yields

$$
\left(I-T_{2} B_{2}\right) y(t)=T_{2}\left(\int_{-\infty}^{t} H_{1}(t, s, x(s)) d s+\int_{t}^{\infty} H_{2}(t, s, x(s)) d s\right) .
$$

Therefore, since $\left\|T_{2} B_{2}\right\| \leq 1-\frac{m}{M}<1$, we obtain

$$
y(t)=\left(I-T_{2} B_{2}\right)^{-1} T_{2}\left(\int_{-\infty}^{t} H_{1}(t, s, x(s)) d s+\int_{t}^{\infty} H_{2}(t, s, x(s)) d s\right) .
$$

Obviously

$$
\begin{aligned}
x(t) & =\int_{-\infty}^{t} f(t, s, x(s+t)) x(s) d s+\int_{t}^{\infty} g(t, s, x(s+t)) d s \\
& +\left(I-T_{2} B_{2}\right)^{-1} T_{2}\left(\int_{-\infty}^{t} H_{1}(t, s, x(s)) d s+\int_{t}^{\infty} H_{2}(t, s, x(s)) d s\right) .
\end{aligned}
$$

It is obvious that $y(t)$ is the unique $T$-periodic solution of 2.6$)$ for $h \in C_{T}^{+}$.

\section{Periodic solutions}

In this section we offer existence criteria for the periodic solutions of the second-order nonlinear neutral integro-differential equations with infinite distributed delay. Lastly in this section we state the Schauder fixed point theorem which enables us to prove the existence of periodic solutions to (1.1) and (1.2). For its proof we refer the reader to [14]. This section is devoted to results concerning the condition (3.2) below. We already know, from Lemma 2.5, that the existence of periodic solutions for (1.1) is equivalent to the existence of solutions for the operator equation $x=D_{1} x$ (3.1), that is, the fixed points in $C_{T}^{J}$ of $D_{1}$. So we assume, for any $J>0$, that there are continuous functions $F_{J}: \triangle^{-} \rightarrow \mathbb{R}^{+}$and $G_{J}: \triangle^{+} \rightarrow \mathbb{R}^{+}$such that

$$
\begin{gathered}
|f(t, s, \varphi)| \leq F_{J}(t, s), \quad(t, s, \varphi) \in \triangle^{-} \times C_{T}^{J}, \\
F_{J}(t+T, s+T)=F_{J}(t, s), \quad(t, s) \in \triangle^{-},
\end{gathered}
$$

and

$$
\begin{gathered}
|g(t, s, \varphi)| \leq G_{J}(t, s), \quad(t, s, \varphi) \in \triangle^{+} \times C_{T}^{J}, \\
G_{J}(t+T, s+T)=G_{J}(t, s), \quad(t, s) \in \triangle^{+} .
\end{gathered}
$$

Whenever necessary, we shall consider

$$
\int_{-\infty}^{t} H_{1}(t, s, \varphi(s)) d s+\int_{t}^{\infty} H_{2}(t, s, \varphi(s)) d s \leq \eta .
$$

We define an operator $D_{1}$ on $C_{T}$ as follows, $\varphi \in C_{T}$ implies that

$$
\left(D_{1} \varphi\right)(t)=\int_{-\infty}^{t} f(t, s, \varphi(s+t)) d s+\int_{t}^{\infty} g(t, s, \varphi(s+t)) d s+\left(P_{1} \varphi\right)(t) .
$$

It is easy to check that $\left(D_{1} \varphi\right)(t+T)=\left(D_{1} \varphi\right)(t)$.

We are now ready to state existence $T$-periodic solution criteria for 1.1)-1.2.

Proposition 3.1. Assume that

$$
\int_{-\infty}^{t-\tau} F_{J}(t, s) d s+\int_{t+\tau}^{\infty} G_{J}(t, s) d s \text { converges to } 0
$$

as $\tau \rightarrow \infty$, uniformly for $t \in \mathbb{R}$. Then, the set $\left\{D_{1} \varphi \mid \varphi \in C_{T}^{J}\right\}$ is equicontinuous. 
Proof. For this purpose, we show that for each $\varepsilon>0$ there is a continuous increasing positive function $\delta=\delta_{J}(\varepsilon):(0, \infty) \rightarrow(0, \infty)$ with

$$
\left|\left(D_{1} \varphi\right)\left(t_{1}\right)-\left(D_{1} \varphi\right)\left(t_{2}\right)\right| \leq \varepsilon, \text { if } \varphi \in C_{T}^{J} \text { and } t_{1}<t_{2}<t_{1}+\delta .
$$

First we prove that there is a continuous increasing positive function $\delta^{-}=\delta_{J}^{-}(\varepsilon):(0, \infty) \rightarrow(0, \infty)$ with

$$
\left|\left(A^{-} \varphi\right)\left(t_{1}\right)-\left(A^{-} \varphi\right)\left(t_{2}\right)\right| \leq \frac{\varepsilon}{3} \text { for } \varphi \in C_{T}^{J} \text { and } t_{1}<t_{2}<t_{1}+\delta^{-},
$$

where $A^{-}$is defined by

$$
\left(A^{-} \varphi\right)(t):=\int_{-\infty}^{t} f(t, s, \varphi(s+t)) d s, t \in \mathbb{R} .
$$

From 3.2 , for any $\varepsilon>0$, there is a $\tau>0$ such that

$$
\left(A^{-} \varphi\right)(t) \leq \int_{-\infty}^{t-\tau} F_{J}(t, s) d s \leq \frac{\varepsilon}{12}, t \in \mathbb{R} .
$$

For any $\varphi \in C_{T}^{J}$ and $t_{1}$ and $t_{2}$ with $t_{1}<t_{2}$, we have

$$
\begin{aligned}
& \left|\left(A^{-} \varphi\right)\left(t_{1}\right)-\left(A^{-} \varphi\right)\left(t_{2}\right)\right| \\
& =\left|\int_{-\infty}^{t_{1}} f\left(t_{1}, s, \varphi(s+t)\right) d s-\int_{-\infty}^{t_{2}} f\left(t_{2}, s, \varphi(s+t)\right) d s\right| \\
& \leq \int_{t_{1}-\tau}^{t_{1}}\left|f\left(t_{1}, s, \varphi(s+t)\right)-f\left(t_{2}, s, \varphi(s+t)\right)\right| d s+\int_{-\infty}^{t_{1}-\tau} F_{J}\left(t_{1}, s\right) d s \\
& +\int_{-\infty}^{t_{1}-\tau} F_{J}\left(t_{2}, s\right) d s+\int_{t_{1}}^{t_{2}} F_{J}\left(t_{2}, s\right) d s \\
& \leq \int_{t_{1}-\tau}^{t_{1}}\left|f\left(t_{1}, s, \varphi(s+t)\right)-f\left(t_{2}, s, \varphi(s+t)\right)\right| d s+\frac{\varepsilon}{6}+\int_{t_{1}}^{t_{2}} F_{J}\left(t_{2}, s\right) d s .
\end{aligned}
$$

Since $f(t, s, \varphi)$ is uniformly continuous on $U=\{(t, s, \varphi) \mid t-\tau \leq s \leq t$, and $|\varphi| \leq J\}$, one can, for the $\varepsilon>0$ previously fixed, find a $\delta_{1}$ such that $\delta_{1} \in(0,1)$ and

$$
\left|f\left(t_{1}, s, \varphi\right)-f\left(t_{2}, s, \varphi\right)\right| \leq \frac{\varepsilon}{12 \tau} \text { if }\left(t_{1}, s, \varphi\right),\left(t_{2}, s, \varphi\right) \in U \text { with }\left|t_{1}-t_{2}\right| \leq \delta_{1} .
$$

Hence, if $t_{1}<t_{2}<t_{1}+\delta_{1}$, then we obtain

$$
\int_{t_{1}-\tau}^{t_{1}}\left|f\left(t_{1}, s, \varphi(s+t)\right)-f\left(t_{2}, s, \varphi(s+t)\right)\right| d s \leq \frac{\varepsilon}{12} .
$$

Now let $l:=\sup \left\{F_{J}\left(t_{2}, s\right) \mid t_{1} \leq s \leq t_{2}\right\}$. Then, for the $\varepsilon$, there is a $\delta_{2}$ such that $0<\delta_{2}<\min \left(\frac{\varepsilon}{l}, 1\right)$ and

$$
\int_{t_{1}}^{t_{2}} F_{J}\left(t_{2}, s\right) d s \leq \frac{\varepsilon}{12} \text { if } t_{1}<t_{2}<t_{1}+\delta_{2}
$$

Thus, letting $\delta_{3}=\min \left(\delta_{1}, \delta_{2}\right)$, we see that 3.4 is valid with $\delta^{-}=\delta_{3}$. Since we may assume that $\delta_{3}(\varepsilon)$ is nondecreasing, we can easily conclude that there is a continuous increasing function $\delta^{-}=\delta_{J}^{-}:(0, \infty) \rightarrow$ $(0, \infty)$ which satisfies 3.4 .

Similarly, one can prove that there is a continuous increasing function $\delta^{+}=\delta_{J}^{+}:(0, \infty) \rightarrow(0, \infty)$ with

$$
\left|\left(A^{+} \varphi\right)\left(t_{1}\right)-\left(A^{+} \varphi\right)\left(t_{2}\right)\right| \leq \frac{\varepsilon}{3} \text { if } t_{1}<t_{2}<t_{1}+\delta^{+},
$$


where $A^{+}$is defined by

$$
\left(A^{+} \varphi\right)(t):=\int_{t}^{\infty} g(t, s, \varphi(s+t)) d s, t \in \mathbb{R} .
$$

Finally, it is clear that there is a continuous increasing function $\delta_{4}=\delta_{4}(\varepsilon):(0, \infty) \rightarrow(0, \infty)$ with

$$
\left|\left(P_{1} \varphi\right)\left(t_{1}\right)-\left(P_{1} \varphi\right)\left(t_{2}\right)\right| \leq \frac{\varepsilon}{3} \text { if } t_{1}<t_{2}<t_{1}+\delta_{4} \text { and } \delta_{4}<T \text {. }
$$

Indeed, note that

$$
H(s)=\int_{-\infty}^{t} H_{1}(t, s, x(s)) d s+\int_{t}^{\infty} H_{2}(t, s, x(s)) d s,
$$

and let $\varepsilon>0$. Choosing $0<\delta_{4}<1$ such that for $t_{1}<s<t_{2}<t_{1}+\delta_{4}$,

$$
\left|K_{1}\left(t_{1}, s\right)-K_{1}\left(t_{2}, s\right)\right| \leq \frac{m \varepsilon}{6 \eta M T}, t_{1}<s<t_{2}<t_{1}+\delta_{4},
$$

and

$$
\left|K_{1}\left(t_{1}, s\right)-K_{1}\left(t_{2}, s+T\right)\right| \leq \frac{m \varepsilon}{6 \eta M}, t_{1}<s<t_{2}<t_{1}+\delta_{4} .
$$

Therefore

$$
\begin{aligned}
& \left|\left(P_{1} \varphi\right)\left(t_{1}\right)-\left(P_{1} \varphi\right)\left(t_{2}\right)\right| \\
& \leq \frac{M}{m}\left|\int_{t_{1}}^{t_{2}} K_{1}\left(t_{1}, s\right)\right| H(s)\left|d s-\int_{t_{1}+T}^{t_{2}+T} K_{1}\left(t_{2}, s\right)\right| H(s) \mid d s d s \\
& +\int_{t_{2}}^{t_{1}+T}\left|K_{1}\left(t_{1}, s\right)-K_{1}\left(t_{2}, s\right)\right||H(s)| d s \mid .
\end{aligned}
$$

Since $H(s+T)=H(s)$, then

$$
\int_{t_{1}+T}^{t_{2}+T} K_{1}\left(t_{2}, s\right) H(s) d s=\int_{t_{1}}^{t_{2}} K_{1}\left(t_{2}, s+T\right) H(s) d s .
$$

Consequently,

$$
\begin{aligned}
& \left|\left(P_{1} \varphi\right)\left(t_{1}\right)-\left(P_{1} \varphi\right)\left(t_{2}\right)\right| \\
& \leq \eta \frac{M}{m} \int_{t_{1}}^{t_{2}}\left|K_{1}\left(t_{1}, s\right)-K_{1}\left(t_{2}, s+T\right)\right| d s \\
& +\eta \frac{M}{m} \int_{t_{2}}^{t_{1}+T}\left|K_{1}\left(t_{1}, s\right)-K_{1}\left(t_{2}, s\right)\right| d s \\
& \leq \frac{\varepsilon}{6}+\frac{\varepsilon}{6}=\frac{\varepsilon}{3} .
\end{aligned}
$$

It now follows, from (3.4) 3.5 and (3.6), that 3.3 holds for $\delta=\min \left(\delta_{4}, \delta^{-}, \delta^{+}\right)$.

Theorem 3.2 (Schauder). [14, p. 31] Let $S$ be a closed convex bounded subset of a Banach space $X$. Assume that $A: S \rightarrow S$ is a compact operator. Then, $A$ has at least one fixed point in $S$.

Theorem 3.3. Let the conditions of Proposition 3.1 holds. Suppose that there is an $\alpha>0$ such that for $\varphi \in C_{T}^{J}$

$$
\int_{-\infty}^{t} H_{1}(t, s, \varphi(s)) d s+\int_{t}^{\infty} H_{2}(t, s, \varphi(s)) d s \leq m \alpha,
$$

and

$$
\int_{-\infty}^{t} F_{J}(t, s) d s+\int_{t}^{\infty} G_{J}(t, s) d s+\alpha \leq J .
$$

Then, 1.1) has a T-periodic solution. 
Proof. First, we see that $C_{T}^{J}$ is a closed convex bounded subset of the Banach space $C_{T}$. Let $\varphi \in C_{T}^{J}$ and consider the map $D_{1}$ on $C_{T}^{J}$. We show that $D_{1}: C_{T}^{J} \rightarrow C_{T}^{J}$. First the equation $\left(D_{1} \varphi\right)(t+T)=\left(D_{1} \varphi\right)(t)$ is not difficult to check. Let $\varphi \in C_{T}^{J}$

$$
\begin{aligned}
& \left|\left(D_{1} \varphi\right)(t)\right| \\
& \leq \int_{-\infty}^{t} F_{J}(t, s) d s+\int_{t}^{\infty} G_{J}(t, s) d s \\
& +\left\|\left(I-T_{1} B_{1}\right)^{-1}\right\| \max \int_{t}^{t+T} K_{1}(t, s)\left[\int_{-\infty}^{s}\left|-H_{1}(s, u, \varphi(u))\right| d u\right. \\
& \left.+\int_{s}^{\infty}\left|-H_{2}(s, u, \varphi(u))\right| d u\right] d s .
\end{aligned}
$$

Therefore, since $\left\|T_{1} B_{1}\right\| \leq 1-\frac{m}{M}<1$ we have $\left\|\left(I-T_{1} B_{1}\right)^{-1}\right\| \leq \frac{1}{1-\left\|T_{1} B_{1}\right\|}$ but $1-\left\|T_{1} B_{1}\right\| \geq 1-1+\frac{m}{M}$ thus $\frac{1}{1-\left\|T_{1} B_{1}\right\|} \leq \frac{M}{m}$. So, from the Lemma 2.4. (3.7) and from what we deduced above, it follows that

$$
\left|\left(D_{1} \varphi\right)(t)\right| \leq \int_{-\infty}^{t} F_{J}(t, s) d s+\int_{t}^{\infty} G_{J}(t, s) d s+\frac{1}{m} m \alpha \leq J .
$$

Remains to show $D_{1}$ is continuous on $C_{T}^{J}$. For that, let $\left\{\varphi_{n}\right\}$ be a sequence of elements in $C_{T}^{J}$ such that $\varphi_{n} \rightarrow \varphi \in C_{T}^{J}$ as $n \rightarrow \infty$. We prove that the sequence $\left(D_{1} \varphi_{n}\right)$ converges to $\left(D_{1} \varphi\right) \in C_{T}^{J}$ as $n \rightarrow \infty$. To see this, let $\tau>0$ be given. Since

$$
\begin{aligned}
& \int_{-\infty}^{t}\left|f\left(t, s, \varphi_{n}\right)\right| d s+\int_{t}^{\infty}\left|g\left(t, s, \varphi_{n}\right)\right| d s \\
& \leq \int_{-\infty}^{t} F_{J}(t, s) d s+\int_{t}^{\infty} G_{J}(t, s) d s \leq J-\alpha,
\end{aligned}
$$

and

$$
\begin{aligned}
& \int_{-\infty}^{t}\left|f\left(t, s, \varphi_{n}\right)\right| d s+\int_{t}^{\infty}\left|g\left(t, s, \varphi_{n}\right)\right| d s \\
& =\int_{-\infty}^{t-\tau}\left|f\left(t, s, \varphi_{n}\right)\right| d s+\int_{t-\tau}^{t}\left|f\left(t, s, \varphi_{n}\right)\right| d s \\
& +\int_{t}^{t+\tau}\left|g\left(t, s, \varphi_{n}\right)\right| d s+\int_{t+\tau}^{\infty}\left|g\left(t, s, \varphi_{n}\right)\right| d s
\end{aligned}
$$

then, for all $n \geq 0$,

$$
\begin{aligned}
& \int_{t-\tau}^{t}\left|f\left(t, s, \varphi_{n}\right)\right| d s+\int_{t}^{t+\tau}\left|g\left(t, s, \varphi_{n}\right)\right| d s \\
& \leq \int_{-\infty}^{t} F_{J}(t, s) d s+\int_{t}^{\infty} G_{J}(t, s) d s \leq J-\alpha .
\end{aligned}
$$

Thus, by the dominated convergence theorem we deduce that

$$
\int_{t-\tau}^{t}\left|f\left(t, s, \varphi_{n}\right)\right| d s+\int_{t}^{t+\tau}\left|g\left(t, s, \varphi_{n}\right)\right| d s \rightarrow \int_{t-\tau}^{t}|f(t, s, \varphi)| d s+\int_{t}^{t+\tau}|g(t, s, \varphi)| d s,
$$

because $f$ and $g$ are continuous. Proposition 3.1 shows that the set $\left\{D_{1} \varphi \mid \varphi \in C_{T}^{J}\right\}$ is equicontinuous and the function $D_{1}$ is continuous. Thus $D_{1}$ is compact operator. The conditions of Schauder's theorem are satisfied and there exists $x \in C_{T}^{J}$ with $D_{1} x=x$. Thus, $x$ is a $T$-periodic solution of (1.1). 
Example 3.4. Consider the following second-order neutral functional integro-differential equations with infinite distributed mixed-delays

$$
\begin{aligned}
& \frac{d^{2}}{d t^{2}}\left(x(t)-\int_{-\infty}^{t} f(t, s, x(s+t)) d s-\int_{t}^{\infty} g(t, s, x(s+t)) d s\right) \\
& =a(t) x(t)-b(t)\left(\int_{-\infty}^{t} p(t, s) h_{1}(x(s)) d s+\int_{t}^{\infty} q(t, s) h_{2}(x(s)) d s\right),
\end{aligned}
$$

where

$$
\begin{aligned}
T & =\pi, a(t)=\frac{1}{4} \sin ^{2}(t)+1, b(t)=0.4 \times\left(\frac{1}{4} \sin ^{2}(t)+1\right), \\
p(t, s) & =e^{s-t}, q(t, s)=e^{t-s}, h_{1}(x)=\sin (x), h_{2}(x)=\cos (x), \\
f(t, s, x) & =0.5 \sin (x), g(t, s, x)=0.2 \cos (x) .
\end{aligned}
$$

Clearly, all the hypotheses of Theorem 3.3 are satisfied. Then 3.9 has a $\pi$-periodic solution.

Using Lemmas 2.9 and 2.10 and reasoning as in last section, it is possible to establish the following theorem for the existence of $T$-periodic solution to 1.2 .

Theorem 3.5. Assume that the hypotheses of Theorem 3.3 hold. Then, (1.2) has a T-periodic solution.

\section{References}

[1] A. Ardjouni, A. Rezaiguia and A. Djoudi, Existence of positive periodic solutions for two types of second-order nonlinear neutral differential equations with infinite distributed delay, J. Appl. Math. Comput. 47 (2015), 291-314.

[2] A. Ardjouni, A. Djoudi, Existence of positive periodic solutions for two types of second order nonlinear neutral differential equations with variable delay, Proyecciones J. Math. 32 (4) (2013), 377-391.

[3] A. Ardjouni, A. Djoudi, Existence of periodic solutions in totally nonlinear neutral dynamic equations with variable delay on a time scale, Math. Eng. Sci. Aerosp. MESA 4(3) (2013), 305-318.

[4] A. Ardjouni, A. Djoudi, Existence of positive periodic solutions for two kinds of nonlinear neutral differential equations with variable delay, Dyn. Continuous Discrete Impulsive Syst. Ser. A Math. Anal. 20 (2013), 357-366.

[5] A. Ardjouni, A. Djoudi, Existence of positive periodic solutions for nonlinear neutral dynamic equations with variable delay on a time scale, Malaya J. Matematik 2 (1) (2013), 60-67.

[6] A. Ardjouni, A. Djoudi, Existence and positivity of solutions for a totally nonlinear neutral periodic differential equation, Miskolc Math. Notes 14(3) (2013), 757-768.

[7] A. Ardjouni, A. Djoudi, Existence of positive periodic solutions for a second-order nonlinear neutral differential equation with variable delay, Adv. Nonlinear Anal. 2013. doi:10.1515/anona-2012-0024.

[8] A. Ardjouni, A. Djoudi, Existence of periodic solutions for a second order nonlinear neutral differential equation with functional delay, Electron. J Qual. Theory Differ. Equ. 2012 (31) (2012), 1-9.

[9] A. Ardjouni, A. Djoudi, Existence of periodic solutions for totally nonlinear neutral differential equations with variable delay, Sarajevo J. Math. 8(1) (2012), 107-117.

[10] A. Ardjouni, A. Djoudi, Existence of positive periodic solutions for a nonlinear neutral differential equation with variable delay, Appl. Math. E-Notes 12(2012), 94-101.

[11] A. Ardjouni, A. Djoudi, Periodic solution in totally nonlinear dynamic equations with functional delay on a time scale, Rend. Sem. Mat. Univ. Politec. Torino 68 (4) (2010), 349-359.

[12] H. Deham, A. Djoudi, Existence of periodic solutions for neutral nonlinear differential equations with variable delay, Electron. J. Differ. Equ. 2010127 (2010), 1-8.

[13] W. S. Cheung, J. Ren and W. Han, Positive periodic solutions of second order neutral functional differential equations, Nonlinear Analysis 71 (2009), pp. 3948-3955.

[14] M. Feckan, Topological degree approach to bifurcation problems (topological fixed point theory and its applications), 2008 Springer Science + Business Media B.V. ISBN 978-1-4020-8723-3.

[15] J. Hale, Theory of functional differential equations, Vol. 3, 2nd edn. Springer-Verlag, New York, Heidelberg, Berlin (1977).

[16] J. K. Hale, S. M. Verduyn Lunel, Introduction to functional differential equations, Vol. 99. Springer-Verlag, New York (1993).

[17] W. Han and J. Ren, Some results on second-order neutral functional differential equations with infinite distributed delay, Nonlinear Anal. 70(2009), 1393-1406.

[18] J. Wu and Z. C. Wang, Two periodic solutions of second-order neutral functional differential equations, Journal of Mathematical Analysis and Applications 329 (2007), 677-689. 